\title{
International Symposium on Experimental Spinal Cord Repair and Regeneration
}

Supported by the 'Associazione per la ricerca sulle lesioni del midollo spinale' (Italian Association for research on spinal cord lesions).

The Symposium will take place on 20th and 21st October 1996, immediately after the Eurospine '96 Meeting in Zurich (15th-19th October):

Registration is free.

This announcement also serves as call for papers.

Provisional registrations are welcome within the end of May 1996

The Organizing Secretariat Studio Progress Snc. ViaC. Cattaneo, 51 1-25121 Brescia (Italia)

Tel: 030/290326, Fax 030/40164 